\title{
The Mental Health Problems of HIV Positive Mothers During Pregnancy and After Giving Birth in Mekelle Hospital
}

\author{
Kahsay Weldeslasie Hailemariam \\ Psychology Department, College of Social Sciences and Languages, Mekelle University, Mekelle, Ethiopia
}

Email address:

kasweld@gmail.com,kaspsyc@yahoo.com

To cite this article:

Kahsay Weldeslasie Hailemariam. The Mental Health Problems of HIV Positive Mothers During Pregnancy and After Giving Birth in Mekelle Hospital. Psychology and Behavioral Sciences. Vol. 4, No. 3, 2015, pp. 132-138. doi: 10.11648/j.pbs.20150403.17

\begin{abstract}
HIV positive pregnant mothers experienced a double burden as a result of having a virus in their blood and the child-bearing. There is high probability of transferring the virus from the pregnant mothers during conception and at the time of giving birth to the child; especially if there is no good care from health professionals and family. Therefore, the intention of the research was to know the psychological distress and subjective burden among mothers during pregnancy and after giving birth. A case study method with cross sectional research design was employed in order to collect the qualitative data from the 30 available pregnant mothers for ART and checkup in the hospital. The mothers were with different family backgrounds, educational level and socio-economic statuses. Mothers faced with different psychological problems like, depression, anxiety, PTSD, stigma and discrimination from their family and the society in general. Mothers were highly worried, depressed and anxious as well as disturbed by the bad memories of their past life experiences while infected by the virus.
\end{abstract}

Keywords: Mental Health Problems, HIV Positive, Post-Partum

\section{Introduction}

HIV positive women of child-bearing age are the fastest growing HIV positive population in the world. As per to the progress reports of the WHO, UNICEF and UNAIDS on HIV/AIDS, about 1.49 million infants every year born with HIV positive [1]. Effective antiretroviral therapy (ART)based protocols for preventing mother-to-child HIV transmission (MTCT) is becoming increasingly accessible worldwide [2]. However, mental health-related factors could potentially undermine its effective delivery and affect the women's quality of life and psychological well-being. For example, depression is a highly prevalent co-morbidity among HIV positive pregnant mothers in general [3] and has been associated with poor HIV viral suppression [4], increased mortality, accelerated disease progression [5] and increased risk of ART non-adherence [6]. Depression among HIV positive mothers has also been correlated with lower scores in the psychological, social, and environmental domains of quality of life [7]. In the case of pregnant HIV positive mothers, such findings give rise to additional concerns, some of which are discussed below.

First, there is a concern that adverse mental health outcomes could affect obstetric and neonatal outcomes, HIV disease management during pregnancy or postpartum and related risk of preventing mother-to-child HIV transmission (MTCT) [8]. Indeed, a recent meta-analysis reported optimal anti-retro viral therapy (ART) adherence in only $76 \%$ pregnant and $53 \%$ postpartum HIV positive mothers worldwide and identified emotional distress, depression, PTSD and substance abuse as barriers to adherence [9]. The meta-analysis highlighted the need for more research and development of appropriate interventions targeting mental health factors among HIV positive pregnant women [9]. However, to the best of the researcher's knowledge, the literature addressing mental health outcomes specifically in pregnant HIV positive mothers has not been synthesized in a manner that would inform development of such interventions.

Treatment guidelines for managing depression during pregnancy jointly developed by the American Psychiatric Association and the American College of Obstetricians and Gynecologists [7] provide extensive overview of relevant literature along with consensus-based treatment and diagnostic algorithms, but do not address HIV-related or HIV-specific clinical factors for pregnant mothers. Next, there is a concern that pregnancy might be a time of 
heightened mental health vulnerability for HIV positive women due to adversary contextual factors, which commonly co-occur with HIV infection among women. For example, three recent literature reviews [9] highlighted the high cooccurrence rates of intimate partner violence (IPV) and HIV infection among women.

One of these three reviews indicated a need to examine the relationship between intimate partner violence (IPV), posttraumatic stress disorder and substance abuse in HIV positive women [3]. Yet, these reviews did not focus on IPV in the context of pregnancy or the post-partum period. Further, a recent comprehensive literature review provided strong evidence of the negative impact of HIV-related stigma on women's participation in MTCT prevention programs in lowincome countries and outlined a theoretical model that includes women's mental health as a variable that might interact with both stigma and preventing mother-to-child HIV transmission (MTCT) [2]. However, the reviews did not discuss the specific mental health-related outcomes like, depression, PTSD, anxiety and other psychosocial problems. For this reason, the researcher conducted the present research.

Ideally, mental health outcomes of pregnant HIV positive women would be evaluated in relation to salient clinical variables, such as HIV disease severity indicators, ART adherence or MTCT risk, as well as to frequently cooccurring contextual factors, such as HIV related stigma; or IPV, which is strongly associated with adverse mental health outcomes in HIV positive mothers [8].

Having HIV in one's blood is obviously distressing by its nature. In addition to that, psychological distress, bearingchild may be another double subjective burden for those mothers especially during pregnancy and after giving birth. That is because a newly born child is highly vulnerable to the transmission of the virus to his/her blood. To reflect the reality, the absence of publications presenting current state of the evidence regarding mental health of HIV positive women during pregnancy remains a gap in the field as well as in Ethiopian context. In order to address this gap, the researcher conducted a comprehensive and detail finding in relation to the global literatures conducted on mental health of pregnant HIV positive women. Clearly speaking, all HIV positive women across the globe may not have the same opportunities and access to key resources necessary for effective HIV care and MTCT. That is why the researcher initiated for conducting the present research based on the existing reality of Mekelle city in particular and Ethiopia in general.

Generally, the research conducted to describe and synthesize the current evidence on the HIV positive childbearing mothers and the extent or correlates of mental health outcomes of pregnant HIV positive mothers and identify clinical as well as research implications of the problem. Specifically, the research conducted for the following purposes;

- To identify adverse psychological problems with mothers who have pregnancy and after giving birth.

- To explain the psychological distress and subjective burdens of mothers during pregnancy and post-partum periods.

- To indicate possible ways for policy makers in order to incorporate strategies that can help mothers during pregnancy and post-partum periods.

- To indicate mental health professionals how to assist mothers providing psychological services.

\section{Method and Design of the Study}

The researcher employed a case study method with cross sectional research design in order to collect detail information from mothers who are from different socioeconomic backgrounds. The case study method helps to collect in-depth and comprehensive information from the participants; whereas, the cross sectional research design also helps to collect diversified information from the diversified participants. The other reason for using this method is also, it is difficult to get many participants at a time in the hospital. Therefore, case study method is more convenient than other methods for participants who are few in number.

\subsection{Research Site}

The research was conducted in Mekelle Hospital, the regional sate of Tigray, Ethiopia. In the hospital above 400 patients with different health problems get treatment services per day. In the city there are few governmental and private hospitals and clinics. Most people preferred to go to the government hospitals and clinics because patients get treatment service with minimum cost in the governmental health institutions. Especially those who are taking antiretroviral therapy and vaccination gain the service for free.

\subsection{Population and Samples of the Study}

In the hospital about 10 mothers gain anti-retroviral therapy and health checkup services in average. For this reason, it is difficult to put the total population since it varies from day to day. Therefore, a researcher selected 30 participants within three consecutive days. Once the researcher was selected the 30 participants, detail information was collected with the assistance of clinical nurses in the hospital. No time limitation was given for each participant during the interview session.

\subsection{Instruments of Data Collection}

The researcher was employed semi-structured interview in order to collect comprehensive and detail information about the mental health outcomes of mothers during pregnancy and after giving birth. The semi-structure interview questions were developed by the researcher reading different previous research results in order to assure the reliability and validity of the instruments. Among the instruments, stigma and discrimination interview questions, PTSD questions, anxiety and depression questions were employed so as to identify the mental health outcome of child-bearing mothers with HIV 
positive.

\subsection{Data Collection Procedures}

The researcher selected participants within three consecutive days. Having selected the participants, the researcher oriented the clinical nurses how to make an interview with the participants. Clinical nurses conducted interview sessions with the mothers in different time intervals After collected enough information that fulfills the objective of the research, the researcher gathered the information from the clinical nurses. Using the clinical nurses as a data collector, the researcher collected valid and reliable information from the participants.

\subsection{Variables of the Study}

Independent variables: Having HIV in child-bearing mothers' blood as well as stigma and discrimination.

Dependent variable: the mental health outcome like, depression, anxiety, PTSD, stigma and discrimination. Stigma and discrimination cab be dependent and independent variable in the finding.

\subsection{Ethical Considerations}

First and foremost, participants' informed consent was assured by the researcher before starting to collect the data. Participants were informed about their rights and duties during the interview sessions by the data collectors. Participants had informed even to stop the interview sessions when the interview goes in a way that exposes their privacy to the public.

\section{Results}

Table 1. Socio-Demographic Information of Participants

\begin{tabular}{llll}
\hline Categories & Components & No. & \% \\
\hline \multirow{4}{*}{ Age } & $20-30$ & 12 & 40 \\
& $31-40$ & 15 & 50 \\
Residence & $41-50$ & 3 & 10 \\
& Rural & 9 & 30 \\
Mothers' status & Urban & 21 & 70 \\
Educational level & Mothers who are giving birth & 18 & 60 \\
& Pregnant mothers & 12 & 40 \\
& Illiterate & 9 & 30 \\
& $1-8$ Grade & 12 & 40 \\
& $9-12$ Grade & 6 & 20 \\
Total & Diploma holder & 3 & 10 \\
& First degree and above & 0 & 0 \\
& & 30 & 100 \\
\hline
\end{tabular}

The above table illustrated that, most of HIV positive mothers in the hospital found between the age ranges of 31 40. That is to mean that, $15(50 \%)$ of mothers visited the hospital for anti-retro viral therapy and health checkup to the hospital per day. This is the most prevalent age range that visits the hospital in that particular place. The ages from $20-$ 30 is the second age group that was highly prevalent in the hospital. It means, $12(40 \%)$ of mothers who are found in the age group between $20-30$ visit the hospital per day for attending anti-retro viral therapy and health checkup to the hospital. The remaining age group (i.e., $41-50$ ) is the least prevalent age group that visited the hospital per day. Mean that, only $3(10 \%)$ of HIV positive mothers who are found between the age group of $41-50$ visited the hospital for antiretro viral and health checkup to the hospital during pregnancy and post partum periods.

The other socio-demographic variable that analyzed in the above table is the mothers' residence place. $21(70 \%)$ of HIV positive mothers come from Mekelle city and other towns which are found around Mekelle city. The remaining $9(30 \%)$ of HIV positive mothers come from the rural districts around Mekelle city for attending anti-retro viral therapy and health checkup. This data shows that, most of mothers who are living in towns and city were highly affected by the virus than rural mothers. The other variable that analyzed in the above table is the HIV positive mothers' status. $18(60 \%)$ of HIV positive mothers were those who are giving birth but attending for anti-retro viral therapy and for vaccination to their children in the hospital. The remaining $12(40 \%)$ HIV positive mothers were pregnant and visit the hospital for vaccination or anti-retro viral therapy.

The last but not least variable is the HIV positive mothers' educational level. 12(40\%) of HIV positive mothers were found in the grade levels of from $1-8$. Those who are illiterate $9(30 \%)$ HIV positive mothers were the second rank who visited the hospital for anti-retro viral and vaccination to the hospital during pregnancy and after giving birth. The remaining from $9-12$ grades and diploma educational levels of HIV positive mothers were the third and fourth ranks respectively that prevalently visited the hospital during pregnancy and after giving birth for anti-retro viral therapy or for vaccinating their children. No first degree and above degree holder HIV positive mothers were visited the hospital during the data collection.

\subsection{The Level of Anxiety and Depression of HIV Positive Mothers During Pregnancy and After Giving Birth}

The HIV positive pregnant and those who were found in the post-partum period of mothers had more or less the same psychological distress and subjective burden. As mothers during the interview sessions explained that, they have high level of anxiety and depression as a result of the virus in their blood and the childbearing. Most of participants during the interview sessions explained that, they prefer to leave the world in order to escape from the sufferings faced in their day to day life. Therefore, this suicidal ideation is a typical symptom of depression. They also reported that, fear; apprehension, worry and tension psychological states disturb their life frequently when they 
think about their future life and the life of their children. They are always in the state of anxiety of transferring the virus to their children during the pregnancy time and while breast feeding to the children.

The most extreme statements that one HIV positive pregnant mother reported during the interview was, "I always think more about my future life and the life of my child. I attempted many times to commit suicide; but when I think about my child's future life, I immediately canceled my decision. Worries and stress makes me meaningless person. I am living in this world for visiting whatever problems given to human beings. Living in this world is meaningless for me". Most of the reports of the participants were more or less similar with this idea.

The finding shows that, more than 18(60\%) of childbearing mothers scored high on the anxiety and depression interview questions as a result of having the virus in their blood. This shows that, childbearing in addition to the HIV in mothers' blood exposed them to more severe anxiety and depression. Most of the mothers had pessimistic attitude towards their future life and the life of their children. "It would better if I were not born or exist in this earth". This is a statement taken from one mother during the interview. Generally, most mothers had very severe level of anxiety and depression from their response. There was no any significant difference in the experience of anxiety and depression as a result of being a pregnant mother or being found in the postpartum period.

\subsection{The PTSD Experience Level of HIV Positive Mothers During Pregnancy and After Giving Birth}

As per to the report of three mothers during the interview, they feel confused when remembering the past events about the infection by the virus. Even they tried to fly somewhere else and to repress the bad memories or the flashback of the bad memories oscillating in their awareness. The obsession thinking of the bad memories impaired their day to day functioning of the mothers during the pregnancy and even after giving birth. As one mother explained that, "I need to fly somewhere else in order to escape from the bad memories oscillating in my awareness. However, when I think deeply, there is no any place that is free from such kind of bad experience". Sometimes I become socially and psychologically very impaired; especially when I remember the distressing past events.

Generally, almost all mothers (pregnant and in the post partum period) had high level of PTSD experience in their daily life. This shows that, having HIV in mothers blood equally distressed and traumatized them at the time of pregnancy and even after giving birth.

\subsection{The Experience of Stigma and Discrimination on HIV Positive Mothers During Pregnancy and After Giving Birth}

The HIV positive mothers were faced with stigma and discrimination from their family and the society in general during the pregnancy and post-partum periods. As one mother reported that, "my elder daughter stigmatized me; she is not voluntary to drink, eat and even to sleep with me. This behavior is observed after I disclosed my diagnosis to my family. When I remember the previous behavior of my daughter, I become very distressed and apprehensive. The stigma and discrimination I receive from my family and the society in my neighbor is very distressing more than the actual problem that I suffering from the virus". In addition to the above report, most participants during the interview expressed that, they experienced more or less similar stigma and discrimination from their families and the society in their neighbors in general during the pregnancy and post-partum periods.

Some mothers reported that, there are many other mothers who do not come to the hospital for fear of disclosing the problem to doctors. These mothers consider as if the problem is disclosed to the doctors, then after everybody may know them and discriminate as well as stigmatized them from the society. For fear of stigma and discrimination, mothers avoid going to hospitals as a result their life expectancy and quality of their life as well as their children's health becomes very low.

To sum up, it is not the presence of the virus in the mothers' blood that makes them very distressed and depressed; rather it is the stigma and discrimination they experienced in their daily lives from the family and their neighbors in general. The most frequent response of the mothers was, they prefer to avoid social lives in order to escape from gossips as well as stigma and discrimination of the society in their neighbor. This shows that, stigma and discrimination is the most stupid social behavior that highly affects the mental health of victims of HIV/AIDS. Therefore, everybody should give love and affection to those who affected by the virus in order to sustain the life expectancy of mothers. In short, the overall of the research findings are explained in the figure below very well; 


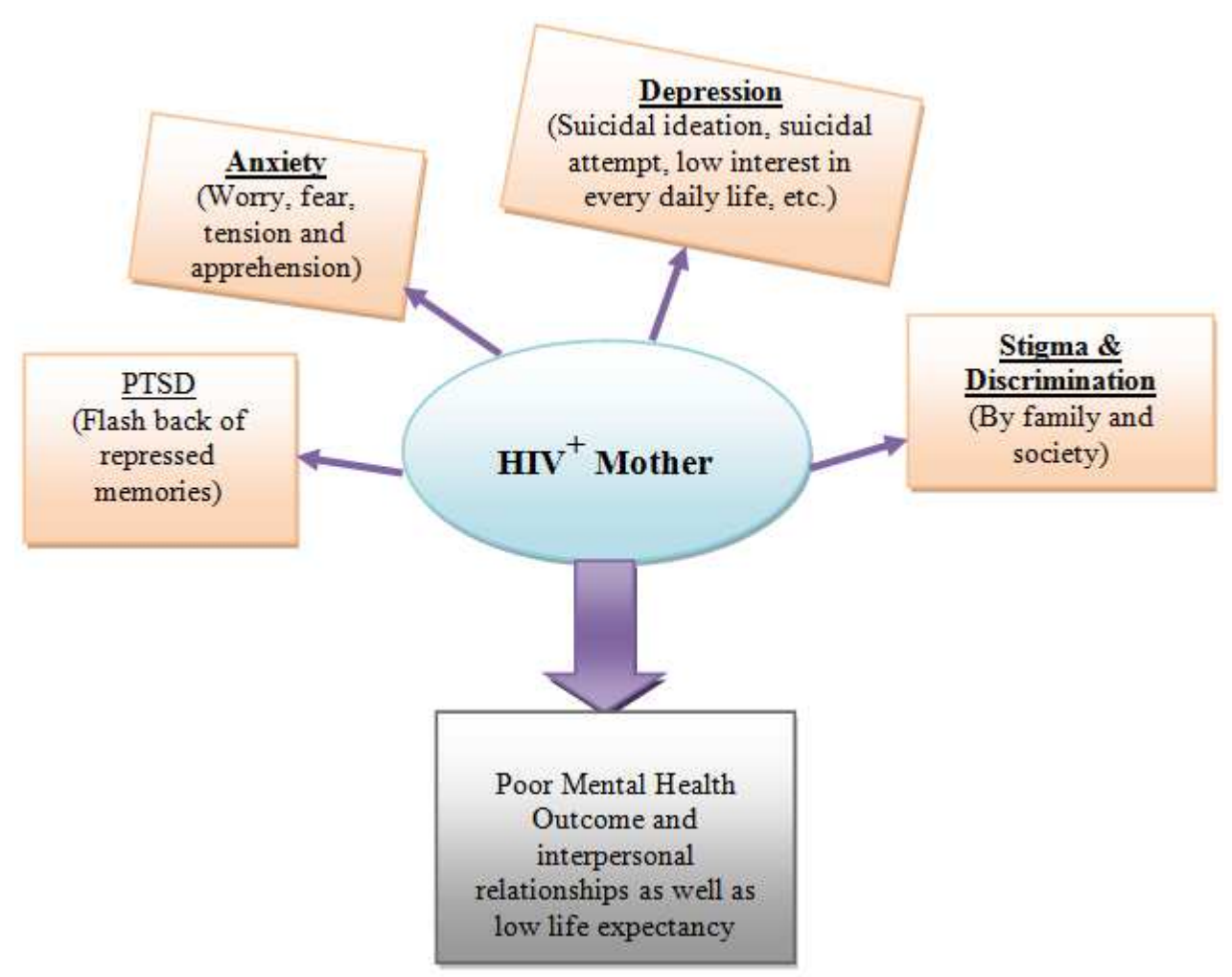

Picture 1. The Conceptual Framework of the Study

\section{Discussions}

\subsection{The Level of Anxiety, Depression and PTSD of HIV Positive Mothers During Pregnancy and After Giving Birth}

The presence of HIV in mothers' blood leads to poor mental health outcome. The major poor mental health outcomes are anxiety, depression, PTSD as well as stigma and discrimination from the society. Different previous findings also supported to the present research finding. High levels of common mental disorders have been identified among people living with HIV/AIDS in communities around the world [10]. There is evidence from low and middle income and high income settings that Common Mental Disorders such as depression, anxiety and PTSD have an adverse impact upon adherence [11], as well as additional negative effects upon HIV-related clinical outcomes such as disease progression and survival, which are independent of the effects of common mental disorder upon adherence [12]. Certain types of sexual harassment or rape experiences qualify as PTSD stressors and that it is reasonable to conclude that traumatic effects (PTSD) flow from the harassment or rape [13].

For instance, $85 \%$ of pregnant HIV positive women from a Zambian urban cohort $(\mathrm{N}=45)$ reported depressive symptoms of "loss of interest in life, ideas of guilt and worthlessness, poor concentration and lethargy"; and recurring thoughts of suicide [14]. Also, HIV positive status was associated with increased risk of antenatal depression in a South African urban cohort $(\mathrm{N}=387)$ [15], added risk and severity of emotional distress in an Angolan cohort $(\mathrm{N}=157)$ [16] and higher odds of increased anxiety.

Although diagnosis and life events related to diagnosis and disclosure undoubtedly contribute to the poor mental health of mothers living with HIV/AIDS [17], there is evidence to suggest that the presence of HIV in the blood may also play a role in producing depression-like symptoms [18]. The majority of research on HIV/AIDS and mental health has been carried out among clinical populations: the time of onset of co-morbid depression and the mechanisms for this are therefore unclear. Although there is evidence to suggest that asymptomatic people living with HIV/AIDS exhibit some cognitive deficits [10], the prevalence of poor cognitive functioning among people in low income settings at an early, preclinical stage has not yet been investigated.

Generally, previous findings show that HIV positive mothers experienced different psychological problems following the diagnosis. Among the psychological problems, deterioration of cognitive functioning, anxiety, depression and PTSD are the major poor mental health outcomes of HIV positive mothers. That is because, pregnancy is a time of heightened mental health vulnerability for HIV positive women due to adversary contextual factors, which commonly co-occur with HIV infection among women [9]. Therefore, the researcher concluded that psychiatric symptoms, particularly depression, and mental health vulnerabilities (e.g., inadequate coping skills) are wide spread among pregnant HIV mothers globally and have a potential to affect psychological well-being, quality of life and salient clinical outcomes. 


\subsection{The Experience of Stigma and Discrimination on HIV Positive Mothers During Pregnancy and After Giving Birth}

Stigma is referred to as a set of prejudicial attitudes, stereotypes, discriminatory behaviors and biased social structures endorsed [19] by sizeable social groups about discredited subgroups. It includes the processes of labeling, separation, stereotyping, status loss, emotional reactions and discrimination in power situations, [19].

HIV positive mothers experience different psycho-social problems from the families and the society in general. Mothers do not go to hospitals for ART fear of disclosing the problem to doctors. HIV positive mothers faced with lack of appropriate psychosocial support due to the virus in their blood. The reason for this may be, due to lack of knowledge about the nature of the illness and the way how the virus transmits from one person to another [20]. Therefore, the government and other concerned bodies should design strategies to improve the range of mental health and psychosocial outcomes in newly HIV-diagnosed pregnant women (i.e., to increase HIV disclosure, self esteem, active coping and positive social support or zero stigma and discriminations, decrease depression, avoidant coping, and negative social support) [20].

Furthermore, a comprehensive literature review provided strong evidence of the negative impact of HIV-related stigma on women's participation in MTCT prevention programs in low-income countries and outlined a theoretical model that includes women's mental health as a variable that might interact with stigma and preventing mother-to-child HIV transmission (MTCT) [2].

\subsection{Limitations of the Study}

The research would better if it were studied in more participants than on 30 individuals only. That is because, the more participants were involved in a research, the more diversified and comprehensive information would collect from the participants. However, there was no any organization that helps the researcher in funding for data collectors and other costs needed for the research. For this reason, the researcher limited the number of participants in to 30 individuals only. The other potential limitation of this study may be some participants were not freely expressing their feelings and emotions. This may be due to the repressive culture of that particular society in general. Therefore, it would better if they were freely expressing their emotions and feelings they had as a result of the virus in their blood. Even though the above problems were faced with during the data collection processes, the researcher tried to solve wisely using convenient research methods and sample selection techniques.

\subsection{Implications of the Study}

The study implies that, HIV positive mothers are suffering from different psycho-social problems like, anxiety, depression, PTSD as well as stigma and discriminations as a result of having the virus in the mothers' blood. For this reason, health professionals and health policy makers should deeply assess best strategies that could help mothers for the positive mental health outcomes. The research also implies that, the presence of the virus in mothers' blood is not the only problem instead; the stigma and discrimination by the society is another head ache to the victims of the virus. Therefore, everybody should create awareness about the nature of the illness and the way of transmission of the virus from person to person on the society using different social Medias. Mothers who were pregnant and those who were giving birth equally experienced the psycho-social problems as a result of the virus in their blood. Thus, health professionals should equally treat HIV positive mothers in the health institutions.

\section{Conclusions}

HIV positive mothers were faced with different psychosocial problems like, anxiety, depression, PTSD as well as stigma and discrimination because of the virus in their blood. This makes double burden to mothers during the pregnancy and even after giving birth. There are money mothers who did not go to hospital fear of disclosing the problem to doctors. This fear of going to doctors may be as a result of fear of stigma and discrimination from the society. Mothers who were pregnant and those who were giving birth equally experienced the psycho-social problems. Mothers score high in the anxiety, depression, and PTSD as well as stigma and discrimination interview questions regardless of being pregnant or giving birth. This shows that the problem is equally distressing by its nature.

\section{Recommendations}

The researcher recommends the following future directions for every concerned body about the problem based on the above conclusions.

- People are stigmatizing and discriminating mothers because of lack of knowledge about the nature of the illness. Therefore, the government or other health professionals should create awareness on the society about the nature and way of transmission of the virus from person to person using different programs.

- There is no any institution in the city that provides psychological services for people who have different psychological problems. For this reason, guidance and counseling service provider institutions should be established in the city.

- Everybody should initiate mothers to go to hospital for ART and other health checkups regularly desensitizing the perceived stigma and discrimination of mothers from the society.

- Mental health researchers should conduct a large scale research upon the area in the future in order to suggest possible strategies of talking the problem. 


\section{Acronyms}

MTCT $=$ Mother to Child Transmission

IPV = Intimate Partner Violence

ART $=$ Anti-Retro Viral Therapy

PTSD $=$ Post-Traumatic Stress Disorder

$\mathrm{HIV}=$ Human Immune Virus

\section{Acknowledgments}

First and for most, I would like to express my heartfelt gratitude to the participants of the study who helped me sharing their feelings and psychological problems freely. Next to that, I would like to say thanks for the clinical nurses who are working in Mekelle hospital for their assistance in the data collection processes. Finally, I would like to express my sincere for my family and friends who helped me giving constructive comments and suggestions during the research work.

\section{References}

[1] Progress Report, (2011). Global HIV/AIDS response: epidemic update and health sector progress towards universal access WHO, UNICEF, UNAIDS. HIV/AIDS 2011; http://www.who.int/hiv/pub/progress_report2011/en/. Accessed 9/5/2013.

[2] Suad K, Priscilla D, Diana N. and Nicholas T. (2014). Mental Health of HIV-Sero-positive Women during Pregnancy and Postpartum Period: A Comprehensive Literature Review. Springer Science and Business Media New York (outside the USA)

[3] Owe-Larsson B, Sall L, Salamon E. \& Allgulander C. (2009). HIV infection and psychiatric illness. Afr J Psychiatry (Johannesburg). 12(2):115-28.

[4] Shacham E, Nurutdinova D, Satyanarayana V, Stamm K. \& Overton E. (2009). Routine screening for depression: identifying a challenge for successful HIV care. AIDS Patient Care STDS 23(11): 949-55.

[5] Dube B, Benton T, Cruess D \& Evans D. (2005). Neuropsychiatric manifestations of HIV infection and AIDS.J Psychiatry Neurosci. 30(4):237-46.

[6] Tucker J, Burnam M, Sherbourne C, Kung F. \& Gifford A. (2003). Substance use and mental health correlates of nonadherence to anti-retroviral medications in a sample of patients with human immunodeficiency virus infection. Am J Med. 114(7): 573-80.

[7] Collins P, Holman A, Freeman M. \& Patel V. (2006). What is the relevance of mental health to HIV/AIDS care and treatment programs in developing countries? A systematic review AIDS. 20(12):1571-82.

[8] Turan J. \& Nyblade L. (2013). HIV-related stigma as a barrier to achievement of global PMTCT and maternal health goals: a review of the evidence. AIDS Behavior. 17(7):2528-39.
[9] Nachega J, Uthman O. \& Anderson J. (2012). Adherence to antiretroviral therapy during and after pregnancy in lowincome, middle-income, and high-income countries: a systematic review and meta-analysis AIDS. 26(16):2039-52.

[10] Rosie M, Vikram P, Melanie A, Priya K, Ramesh P, Savio R. \& Martin P. (2013). Symptoms of common mental disorder and cognitive associations with sero-positivity among a cohort of people coming for testing for HIV/AIDS in Goa, India: a cross-sectional survey. Londen, UK: Health Service \& Population Research Department, Institute of Psychiatry, Kings College. Kings College London, London, UK.BMC Public Health publication.

[11] Peltzer K, Friend-du Preez N, Ramlagan S. \& Anderson J. (2010). Antiretroviral treatment adherence among HIV patients in KwaZulu-Natal, South Africa. BMC Public Health, 10:111.

[12] Antelman G, Kaaya S, Wei R, Mbwambo J, Msamanga G, Fawzi W. \& Fawzi M. (2007). Depressive symptoms increase risk of HIV disease progression and mortality among women in Tanzania. J Acquire Immune Deficit Syndrome, 44(4):470477.

[13] Margaret S, Logan E. \& Rebecca W. (2008). Sexual Harassment and Posttraumatic Stress Disorder: Damages beyond Prior Abuse. American Psychology-Law Society/Division 41 of the American Psychological Association

[14] Kwalombota M. (2002). The effect of pregnancy in HIVinfected women. AIDS Care. 14(3):431-3.

[15] Manikkam L. \& Burns J. (2012). Antenatal depression and its risk factors: an urban prevalence study in KwaZulu-Natal. S Afr Med J. 102(12):940-4.

[16] Bernatsky S, Souza R. \& De Jong K. (2007). Mental health in HIV-positive pregnant women: results from Angola. AIDS Care.19 (5): 674-6.

[17] Steward W, Chandy S, Singh G, Panicker S, Osmand T, Heylen E \& Ekstrand M. (2011) Depression is not an inevitable outcome of disclosure avoidance: HIV stigma and mental health in a cohort of HIV-infected individuals from Southern India. Psychological Health Med, 16(1):74-85.

[18] Lawson M, Kelley K. \& Dantzer R. (2011). Intra-cerebroventricular administration of HIV-1 That induces brain cytokine and indoleamine 2,3-dioxygenase expression: a possible mechanism for AIDS co-morbid depression. Brain BehavImmun, 25(8):1569-1575.

[19] Kahsay W. The Psychological Distress, Subjective Burden and Affiliate Stigma among Caregivers of People with Mental Illness in Amanuel Specialized Mental Hospital. American Journal of Applied Psychology. Vol. 4, No. 2, 2015, pp. 33-47. doi: 10.11648/j.ajap.20150402.13

[20] Mundell J, Visser M. \& Makin J. (2011). The impact of structured support groups for pregnant South African women recently diagnosed HIV positive. Women Health. 51(6):54665 